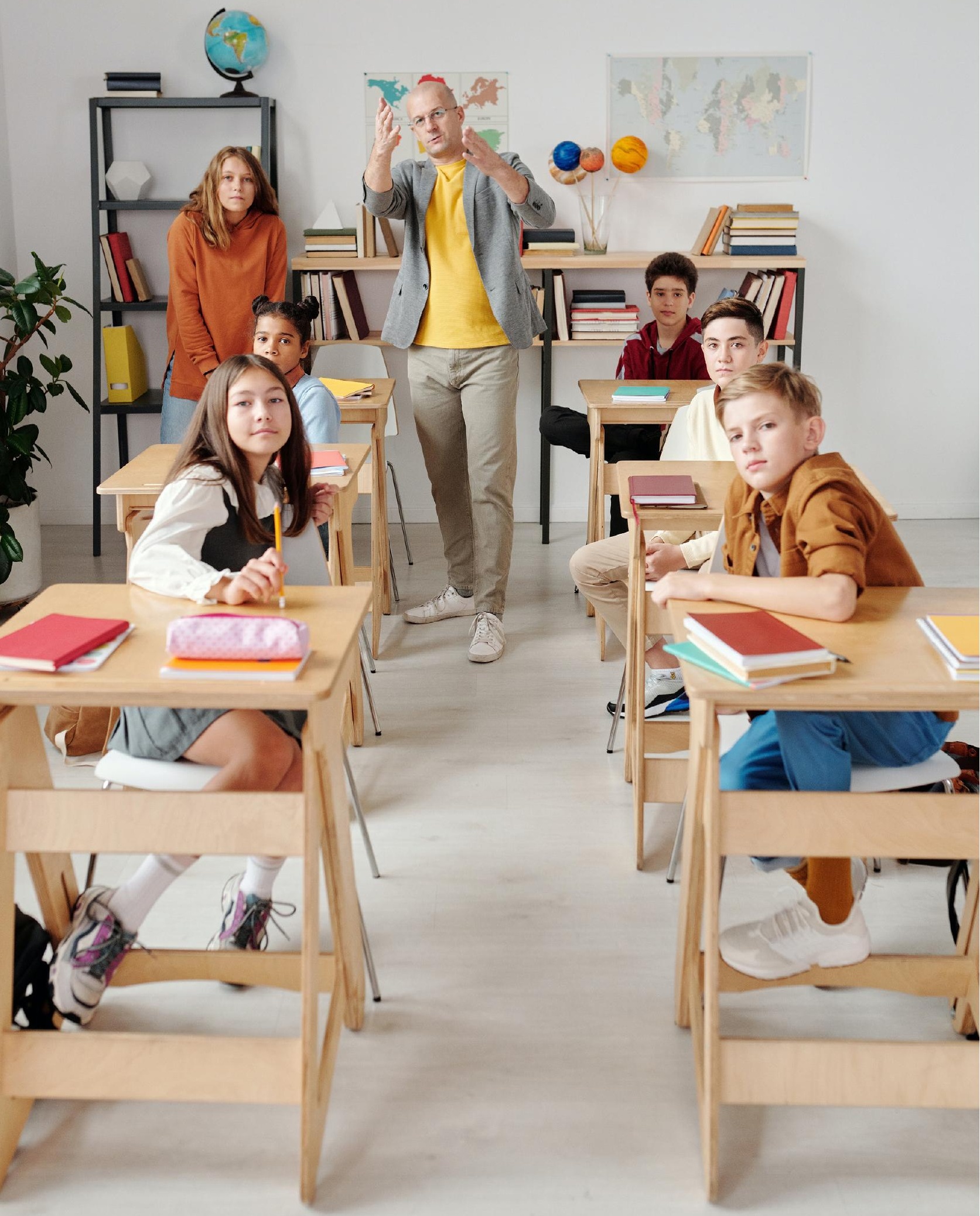




\title{
AMBIENTALIZACIÓN CURRICULAR: UNA EXPLORACIÓN EN LA CONCIENCIA AMBIENTAL
}

\section{Curricular environmentalization: an exploration in environmental awareness}

\author{
Yeymmy Paola Jiménez Gutiérrez ${ }^{1}$, Jaime Ricardo Cristancho Chinome ${ }^{2}$ \\ ${ }^{1}$ Secretaría de Educación de Boyacá, ${ }^{2}$ Universidad Pedagógica y Tecnológica de \\ Colombia Email: ${ }^{1}$ yeymmy.jimenez@uptc.edu.co, ${ }^{2}$ jaime.cristancho@uptc.edu.co
}

(Recibido el 25 de mayo de 2021 - Aceptado el 08 de junio de 2021)

\begin{abstract}
Resumen
La ambientalización curricular como componente transversal incluida en el currículo, que se entiende como una convergencia de saberes, experiencias, objetivos y prácticas metodológicas direccionadoras, presenta un gran desafío para las autoridades educativas y la comunidad académica en general. El presente artículo reflexiona desde bases literarias apoyadas en dos ejes: el eje ambiental y el eje curricular, y en cómo se ha aplicado este proceso dentro del currículo, tomando como referencia diferentes artículos, para finalmente analizar la propuesta realizada por Parga (2013) bajo los pasos de diseño curricular propuestos por Taba (1974) en la realidad nacional. Finalmente, se realizan consideraciones haciendo énfasis en el urgente compromiso que deben tener de las instituciones educativas en la construcción, planeación y ejecución de un currículo ambientalizado y contextualizado.
\end{abstract}

Palabras clave: ambientalización curricular, sostenibilidad, desarrollo sostenible, currículo, educación ambiental.

\begin{abstract}
Curricular environmentalization represents a transversal pedagogic approach included in the curriculum, which is understood as a convergence of knowledge, experiences, objectives, and guiding factor upon methodological practices. It introduces a great challenge for educational authorities and the academic community in general. The present article reflects from literary bases supported by two axes: environmental and curricular centre, on how this process has been applied within the curriculum and taking as a reference the research proposal made by Parga (2013) under steps of curricular design proposed by (Taba, 1974) in the national reality. Finally, considerations are
\end{abstract}


made emphasizing the urgent commitment that educational institutions must have in the construction, planning, and execution of an environmentalized and contextualized curriculum.

Keywords: curricular environmentalization, sustainability, sustainable development, curriculum, environmental education.

La ambientalización curricular como una herramienta pedagógica, pretende la sostenibilidad ambiental y prácticas curriculares, mediante una metodología transversal (Piñero et al., 2017). La vorágine de la globalización y la industrialización de la educación en Colombia (Duque, 2001) ha promovido la cultura de la no ambientalización de la educación, creando currículos estructurales (Díaz-Barriga, 1985 y Sacristán, 1991) que no muestran la flexibilidad, consciencia, y sostenibilidad ambiental necesaria para lograr objetivos de desarrollo sostenible reales dentro del marco de la educación colombiana y su inevitable inclusión en las prácticas globales empresariales de los grandes monopolios económicos y políticos que dominan los diseños y planeaciones curriculares en el país.

La integración de prácticas ambientales que conlleven a propiciar desarrollo sostenible en las poblaciones escolares de los diferentes contextos educativos en el país se muestra como un objetivo prominente que nace desde la literatura y la necesidad de conservar el medio ambiente haciendo énfasis transversal en los diferentes núcleos de todas las áreas que componen la macro-unidad de planeación conocida como currículo (Caicedo y Calderón, 2016). En este sentido, el Ministerio Nacional de Educación de Colombia ha promovido prácticas ambientales en los Colegios nacionales a través de la aplicación del PRAE (Proyecto Ambiental Escolar), el cual tiene como objetivo primario "El desarrollo de actividades donde el estudiante comparta sus ideas a través de experiencias y se conciencien hacia el estudio, visión y conservación del ambiente, dentro de un marco de un desarrollo sostenible" (p. 33). Lo anterior, definido desde la política educativa de cada institución educativa, siguiendo los lineamientos y documentación oficiales que el Ministerio de Educación reglamenta en materia educativa (ver DBA ${ }^{1}$ de Ciencias Naturales, 2018). Sin embargo, considerando los planteamientos de Holguín (2007), algunos colegios les cuesta trabajo la realización de dichos proyectos pues están siendo desarrollados por una sola área de estudios, sin trazarse objetivos trasversales específicos.

Así mismo, Burgos (2017), afirma que los PRAE se han realizado como actividades independientes y de forma fragmentada, igualmente, Garzón y Acuña (2016), indican que

${ }^{1}$ DBA hace referencia a los Derechos Básicos de Aprendizaje, reglamentados por el Ministerio Nacional de Educación de Colombia. La versión más reciente se dio en 2018. 
se ha trabajando con una serie de actividades que no instauran relaciones con el plan de estudios ni forman competencias educativas que permitan responder de forma práctica a los problemas ambientales contemporáneos. Además, Martín y Bayona (2018), consideran que las Instituciones Educativas de Colombia afrontan problemas con la ambientalización curricular, en tanto que los Proyectos Educativos Institucionales (PEI) están descontextualizados de las dinámicas ambientales actuales, como también se carece de equipos de trabajo con un desempeño y rendimiento laboral óptimo.

Ahora bien, la ambientalización del currículo en los ambientes escolares pretende lograr una sostenibilidad de las prácticas ambientales desarrolladas por los estudiantes, y en general, por la comunidad educativa, que apunta a un desarrollo sostenible escolar. Estos dos términos serán repasados desde las concepciones literarias de diferentes autores y serán acercados a la realidad educativa colombiana, proponiendo un posible impacto positivo en la conciencia ambiental (Jiménez y Lafuente, 2007) de las comunidades educativas en Colombia.

Finalmente, este artículo de carácter reflexivo presenta una estructura metodológica basada en dos constructos principales : EJE AMBIENTAL y EJE CURRICULAR, los cuales serán considerados desde una revisión literaria y conceptual, para luego discutir dichos ejes bajo un punto de vista crítico a la luz de evidencia aportada por las diferentes propuestas y proyectos consultados, los cuales han trabajado la Ambientalización Curricular en contextos diversos tales como el escolar, el universitario y el profesional - entendido como una pedagogía multidisciplinar que abarca las diferentes áreas del conocimiento, y que no se limita únicamente a el área de ciencias naturales o ambientales- con el fin de identificar el impacto de dichas propuestas y proyectos, y encaminado a propuestas futuras de investigación.

A continuación, se realizará una revisión de la literatura dentro de los dos constructos principales propuestos para este documento iniciando con el EJE AMBIENTAL.

\section{Eje Ambiental}

En primer lugar, se abordará el concepto de Ambientalización enfocado al contexto académico. En segundo lugar, se revisará la definición de Sostenibilidad como pilar y fundamento de la consciencia ambiental que los educadores promueven dentro de sus estudiantes y de la comunidad académica en general, en el mismo, se revisará la importancia de la sostenibilidad dentro del proceso del desarrollo sostenible. Finalmente, se hará una revisión sobre la educación ambiental. 


\section{Ambientalización}

La ambientalización se puede entender como la inclusión de competencias y capacidades en y hacia los estudiantes encaminada a potenciar sus fortalezas sociales y cognitivas que relacionan directamente procesos de reflexión sobre componentes ecológicos, procesos educativos y de aprendizaje (Meyer, 1998 y Novo, 1998). Por norma general de carácter metodológico y sintáctico, la ambientalización encierra un conjunto de prácticas y saberes transmitidos hacia los aprendices, generalmente enfocados a potenciar sus competencias ambientales (Cañal, 2011).

Entendido de esta forma, la ambientalización y el currículo forman una dicotomía pedagógica y práxica ${ }^{2}$, se encuentra el término Ambientalización Curricular; la ambientalización curricular definida por Piñero et al. (2007) se entiende como "Una potente herramienta para que la formación del alumnado universitario contemple los contenidos necesarios para capacitarles en el desarrollo de sus actividades profesionales desde criterios éticos de responsabilidad hacia el desarrollo sostenible" (p. 2). Aquí se incluye como elemento relevante los criterios éticos y la axiología del currículo que se presenta como componente facilitador en la transversalidad de la ambientalización de los entornos universitarios referenciados por este grupo de autores.

Así mismo, para Junyent et al. (2003), la ambientalización curricular se define como:

Un proceso continuo de producción cultural tendiente a la formación de profesionales comprometidos con la búsqueda permanente de las mejores relaciones posibles entre la sociedad y la naturaleza, atendiendo a los valores de la justicia, la solidaridad y la equidad, aplicando los principios éticos universalmente reconocidos y el respeto a las diversidades. (p. 763)

Se puede observar que este grupo de autores complementa la definición expuesta en Piñero et al. (2007) en términos axiológicos y la amplía hacia la equidad universal. Concepto que se relaciona directamente con la sostenibilidad en términos de aplicabilidad enfocada hacia el desarrollo sostenible. La ambientalización vista como un cambio, como una "Transformación en la visión que el ser humano tiene del universo" (Gallegos, 2017, p. 157). Abarca aspectos ambientales y curriculares, cambios en las políticas y en los diseños curriculares, con el fin de propiciar cambios estables y progresivos hacia una sostenibilidad y un desarrollo sostenible real (Kitzmann et al., 2012).

${ }^{2}$ El término práxica es una adaptación realizada por la autora, la cual define la parte metodológica y práctica en uno solo. 
Finalmente, el concepto global de Ambientalización curricular, citado anteriormente y sintetizado por Campos y Frenk (2010), se puede definir como el desarrollo cultural que busca la mejora de la sociedad y de la naturaleza, partiendo de valores como la justicia, la equidad y la solidaridad. Una visión integradora que se presenta como pilar de desarrollo al proceso de la sostenibilidad, el cual será teorizado a continuación.

\section{Sostenibilidad}

Desde una definición cronológica del término sostenibilidad, se puede mencionar que el informe Brundtland ${ }^{3}$ la define como la concepción que los seres humanos deben tener sobre su planeta, no como una fuente inagotable de recursos, sino como una riqueza que debe ser protegida y constantemente renovada. En esta línea, autores como Pierri (2001), enfocan el concepto hacia el mantenimiento de la base de recursos ambientales con los que cuenta una sociedad, evitando así, el crecimiento desbordado de la economía salvaje, que configura una destrucción masiva de los ambientes naturales y sociales del mundo moderno.

La sostenibilidad se entiende como la responsabilidad estratégica del ser humano con sus recursos naturales, el cuidado y la conservación de estos como fuentes limitadas, de carácter renovable en la medida en que se preserven y se protejan de prácticas industrializadas poco responsables. En esta línea, autores como Estévez (2013), definen la sostenibilidad como un proceso de atender a las necesidades actuales sin comprometer la capacidad de las generaciones futuras de satisfacer las suyas, garantizando el equilibrio entre crecimiento económico, cuidado del medio ambiente y bienestar social. Una definición centrada en el componente de estabilidad, y de proyección hacia el futuro bajo un modelo de conservación de los recursos ambientales, económicos y sociales.

Finalmente, el concepto de sostenibilidad, enfocado hacia la parte ambiental es considerado en la literatura de Arriols (2018), quien expresa que es una consecuencia necesaria, esto debido a las malas prácticas desarrolladas por los gobiernos, compañías, e incluso por las mismas instituciones formadoras, que no consideran a la ambientalización curricular como un objetivo primario transversal dentro de la configuración curricular. Un buen ejemplo citado por el mismo autor refiere a la sostenibilidad implementada en el sector turístico, en el ecoturismo, mediante prácticas responsables, la actividad se desarrolla preservando y protegiendo el recurso natural como fuente de ingreso, como fuente de trabajo, y como capital ambiental perdurable a largo plazo.

${ }^{3}$ El Informe Brundtland, es un informe que enfrenta y contrasta la postura del desarrollo económico actual junto con el de sostenibilidad ambiental; fue creado por la Doctora noruega Gro Harlem Brundtland para las naciones unidas en 1987

Revista ingeniería, Investigación y Desarrollo (I2+D) Vol. 20 - No. 2, 2020 


\section{Desarrollo Sostenible}

En el marco de las prácticas y políticas ambientales responsable y sostenibles, surge el concepto de desarrollo sostenible, el cual se enfoca de acuerdo con el reporte Brundtland (1987), en satisfacer las necesidades actuales sin comprometer la capacidad de las próximas generaciones para satisfacer las suyas propias. Una definición muy cercana a la expresada por Estévez (2013), en cuanto a sostenibilidad ambiental y la capacidad que tienen los países, las personas y las instituciones para proteger los recursos ambientales de forma responsable en el uso y en la planeación.

El desarrollo sostenible como etapa final de un proceso de planeación, tiene unas características específicas, las cuales son citadas por el EO/4:

- Promueve la autosuficiencia regional: la autonomía de las regiones y de las instituciones para planear medidas responsables y cumplibles en términos de conservación ambiental.

- Promueve la autosuficiencia regional: la independencia en medidas focalizadas a atender las problemáticas ambientales y la sostenibilidad.

- Reconoce la importancia de la naturaleza para el bienestar humano: como recurso fundamental proclive a ser protegido y utilizado de forma responsable y eficiente.

- Asegura que la actividad económica mejore la calidad de vida de todos, no solo de unos pocos sectores: que sea ecuánime en términos de cobertura y de calidad dentro de las comunidades.

Desde estos principios de gestión en un programa de Desarrollo Sostenible, se presenta la Educación Ambiental, como puesta en marcha de los planes y proyectos de desarrollo sostenible adaptado a los currículos y ambientado en las aulas de clase.

\section{Educación Ambiental}

Definida por autores como Oliveira et al. (2000), conceptualizan sobre la Educación Ambiental como un proceso amable con las comunidades en términos de permitir sus contribuciones a políticas y proyectos ambientales, mediante la resolución de problemáticas y la toma de medidas encaminadas a proteger los recursos ambientales. Las comunidades académicas en particular tendrían la oportunidad de actuar a favor de los ambientes naturales dentro de procesos y proyectos responsables que potencien su protección y su desarrollo sostenible.

${ }^{4}$ EOI se refiere a la Escuela de Organización Industrial, en su blog del Master Executive en Gestión de las Comunicaciones y Tecnologías de la Información, aclara el concepto de Desarrollo Sostenible. (Velazco, 2013) 
Así mismo, otros autores como Foladori (2000), enfocan su definición de Educación Ambiental hacia los componentes Ecológicos, que son aquellos que consideran y enfocan sus esfuerzos y temáticas a proteger el medio ambiente natural. En segundo lugar, hacia el componente Humano, su relación con los componentes políticos, sociales y económicos encaminados a la protección del recurso humano en un contexto ambiental sostenible.

En otro concepto, autores como Oraison (2000), conciben la educación ambiental como "Una formación integral, tanto a nivel colectivo como individual, con el fin de construir una sociedad más respetuosa hacia las personas y hacia la propia naturaleza que constituye el entorno de la sociedad humana" (p. 25). Estos autores al igual que otro grupo significativo de ambientalistas coinciden en conceptos de integralidad, responsabilidad, cuidado y planeación de políticas responsables y efectivas en términos de aplicabilidad, tanto en contextos académicos, sociales, económicos e incluso axiológicos. Bajo estas premisas, se repasará desde la literatura las concepciones de currículo desde un breve rastreo cronológico, donde se enfocará hacia el currículo de la educación ambiental, enfocado a dar un sustento teórico para iniciar la reflexión sobre los procesos de ambientalización curricular.

\section{EJE CURRICULAR}

\section{Currículo}

Dentro de las concepciones semánticas y prácticas del término currículo, desde una perspectiva cronológica tendiente a dinamizar la reflexión que se realiza en el presente documento, se citan las primeras definiciones documentadas del currículo desde la perspectiva de Bobbit (1918), quien definió el currículo como un paralelo de experiencias encaminadas a enriquecer el desarrollo empírico de una persona, en el cual se le da un entrenamiento expedito encaminado a completar y perfeccionar tareas específicas.

Algunos años después, Campbell y Caswell (1935), enmarcaron al currículo como un conjunto de vivencias y saberes que los aprendices desarrollan desde la orientación de la figura escolar. Entre tanto, con el pasar de los años y las nuevas corrientes ideológicas y metodológicas en el contexto escolar, autores como Inlow (1966), propusieron que el currículo se entendía como "El esfuerzo conjunto y planificado de toda la escuela, destinado a conducir el aprendizaje de los alumnos hacia los resultados de aprendizajes predeterminados" (p. 7). En esta nueva concepción del currículo se encontraron diferencias significativas en cuanto a la conformación e integración de elementos que constituyen al currículo.

Un año después, el pedagogo Estadounidense Johnson (1967), ajustaba la definición expresada por Inlow en los siguientes términos: 
En vista de las deficiencias de la definición popular actual diremos aquí que el currículo es una serie estructurada de objetivos del aprendizaje que se aspira a lograr. El currículo prescribe (o por lo menos anticipa) los resultados de la instrucción. (p. 130)

Aquí se denota una mayor sintetización del concepto de currículo, acercándolo más a las actuales definiciones, en donde se incluyen nuevos componentes como "las competencias", las cuales inicialmente fueron definidas como una destreza, una habilidad específica (en el caso de las ciencias ambientales, las capacidades del individuo para controlar sus actuares y proyectos en pro de la conservación y el uso responsable del medio ambiente que lo rodea ${ }^{5}$ ), la cual debería idealmente ser potenciada en su totalidad antes de avanzar hacia el aprendizaje de otra nueva competencia (Roegiers, 2007). En estos términos, autores como Malagón-Plata (2007) en Pinar (2014), conceptualizan al currículo como "Una oportunidad para construir conexiones más profundas entre el ser humano, la universidad y la sociedad a un nivel más dinámico, integral y sobretodo... relevante" (p. 146). Ahora bien, desde la literatura se entiende el currículo como una macro-integración de saberes, experiencias, contenidos, metodologías, competencias, objetivos, proyectos y políticas que convergen en un interés primario que describe la misión y la visión del educador en su rol de transformador social.

\section{Currículo Ambiental}

La educación ambiental entendida como herramienta de cambio y reflexión de las prácticas modernas de los seres humanos promovidas desde el ámbito académico reflejada hacia la conservación y protección del medio ambiente, mediante planes, proyectos, acciones y experiencias pedagógicas. Entendida de esta forma, la educación ambiental incluye al currículo como macro unidad integradora que va encaminada a potenciar las competencias básicas de los estudiantes en el área de ciencias. Autores como Besciano et al. (2010) creen que la educación ambiental y el currículo no se presentan de forma estática; sino que, "Evolucionan de forma paralela a como lo hace la idea de medio ambiente y la percepción que se tiene de éstos" (p. 6).

Así mismo, en el currículo de la educación ambiental, desde su función creadora y como sustento del proceso escolar, autores como Bedoy (2000), citado por Bresciano et al. (2010) creen que, hoy en día, se hace relevante diseñar los currículos contemplando las dimensiones socioculturales y económicas de los aprendices, con el fin de lograr descifrar y aprovechar las conexiones entre el ser humano y el entorno (entendido como contexto ambiental) que lo rodea. Esta debe dirigirse a toda la humanidad (Wood y Walton, 1990) porque es un desafío para las instituciones formadoras, dentro de la transversalidad

${ }^{5}$ Definición aportada por la autora. 
de las áreas académicas. El currículo ambiental debe integrar políticas y proyectos que propicien cambios en la calidad y estilo de vida de los seres humanos, de sus conductas en frente de sus recursos ambientales, y de sus procesos de interacción con sus semejantes (Febres y Florián, 2002), esto encaminado a la preservación y protección a nivel global de los ambientes sociales, económicos, y en general, naturales.

\section{REFLEXIÓN}

Para el proceso de reflexión, en búsqueda de un análisis realizado desde los elementos constitutivos según Taba (1974, citada en Chablé y Delgado, 2010) de una propuesta de Ambientalización Curricular de la siguiente manera:

-Una declaración de las finalidades y objetivos específicos.

-Una selección y organización del contenido.

-Normas de enseñanza y aprendizaje.

-Programa de evaluación de los resultados (pp. 56-59).

Así mismo, después de analizar los proyectos consultados para el presente documento, se realizará un análisis en luz de los referentes teóricos consultados, con el fin de aterrizar la discusión y dar conclusiones de su aplicabilidad a nivel nacional, y del impacto que esta presenta para la comunidad docente, como actores de cambio.

Para tal ejercicio se consultaron diversos proyectos a nivel nacional e internacional tales como: Ambientalización Curricular en la Educación Superior: Un Estudio Cualitativo de las Ideas del Profesorado, realizado por Mora (2012). En segundo lugar, se consultó una propuesta investigativa a nivel local llamada Propuesta para la Inclusión del Componente Ambiental en el currículo del Pregrado de la UPTC, realizada por Pedraza (2017) A nivel internacional se consultaron 2 proyectos de ambientalización curricular, el primero en Argentina, llamado Proyecto de Ambientalización Curricular en la Universidad Nacional del Nordeste, en el año 2007. En segundo lugar, una tesis doctoral en España llamada La Ambientalización Curricular en las Humanidades, realizada por Arias (2015).

Así mismo, después de realizar una consulta de los referentes teóricos, metodológicos y epistemológicos de los proyectos consultados, se decidió enfocar el proceso de reflexión a manera exploratoria ${ }^{6}$ en el proyecto Un Currículo Ambientalizado para Formar Profesores de Química, realizado por Parga (2013), esto debido al enfoque investigativo

6Exploración, entendida como una revisión de los elementos curriculares propuestos por Taba (1974) citada en Chablé y Delgado (2010). 
de la autora de este texto en el área de la química y las ciencias aplicadas a nivel escolar. Adicionalmente, por el direccionamiento que se dio al trabajo por parte de Parga, en cuanto a la transversalidad (desde el eje ambiental) que posee la enseñanza de la química en el currículo entendido como un proceso de ambientalización, no solo en el profesorado, sino en la comunidad académica en general.

En cuanto a la declaración de las finalidades y objetivos específicos (Taba, 1974), la propuesta desarrollada por Parga (2013) presenta una pregunta específica a ser resuelta, en términos de ambientalización curricular y de inclusión ambiental en la formación de licenciados en ciencias en Colombia. Dicha pregunta de investigación enfoca claramente el proceso de ambientalización curricular, busca una transversalidad del componente ambiental y apunta a un desarrollo sostenible (ver Brundtland, 1987) en el ámbito internacional, nacional y local, con el fin de establecer e identificar los componentes propios del proceso de ambientalización curricular (ver Piñero et al., 2007).

En segundo lugar, esta propuesta cumple con el siguiente momento al realizar una selección y organización del contenido (Taba, 1974). En este caso, enfocado hacia la formación de licenciados en ciencias (incluye química, física y biología) sustentada en una revisión literaria robusta. Así mismo, se establecen propuestas enfocadas hacia la enseñanza y el aprendizaje (Taba, 1974) desde el desarrollo sostenible y la educación ambiental, donde según Parga (2013), “El trabajo se enmarca como estudio cualitativo y en un estudio de caso precedida de un estudio exploratorio de tipo bibliométrico de carácter cualitativo para examinar dónde se ha aplicado y quiénes lo han hecho", (p. 4).

Finalmente, se estableció un programa de evaluación de los resultados (Taba, 1974) desde la metodología; en primer lugar, haciendo un análisis documental de las propuestas curriculares en química en el ámbito nacional. En segundo lugar, mediante un diagnóstico en el profesorado de las licenciaturas a través de una propuesta transversal en aspectos ambientales, la cual generará impactos importantes a nivel local, y nacional. La cual será evaluada y analizada desde su efecto en los participantes.

La anterior propuesta fue tomada como referente para realizar un proceso reflexivo de carácter narrativo en referencia a la aplicación, implementación y expansión de la Ambientalización curricular en Colombia. Si bien es cierto que se han explorado referentes teóricos que incluyen concepciones a nivel global (internacional), se presentaba como elemento relevante el análisis de propuestas a nivel nacional, las cuales fortalecieran el entender de la ambientalización curricular como un proceso transversal sólido, claro y sostenible. 
La sostenibilidad de propuestas de modificación curricular, enmarcadas en el ámbito de la educación nacional, mediada por las diferentes políticas y factores que la condicionan en muchas ocasiones a limitar los currículos a una no-transversalidad ambiental, privando a los aprendices de la adquisición y fortalecimiento de sus competencias ambientales (ver Cañal, 2011), se presenta como un reto académico, social y político (ver Febres y Florián, 2002), en el cual los encargados de los cambios sociales, a través de propuestas, proyectos y metodologías ambientales (los docentes, los directivos en primer lugar) tienen como deber asumir un liderazgo social y académico, encaminado a la transformación de los escenarios de ambientalización curricular actuales.

El cuidado del recurso ambiental como eje del desarrollo académico, social, político y económico (ver Estévez, 2013) en procesos de planeación responsables, inclusivos y productivos que dinamicen el proceso de la ambientalización curricular en escenarios mediados por la educación ambiental, por los proyectos ambientales como el PRAE, por las políticas misionales de cada institución, tanto de educación superior como de educación media y básica. Las cuales, serán determinadas, diseñadas y adoptadas por el Ministerio Nacional de Educación de Colombia, como ente rector y direccionador de las políticas de alto impacto a nivel nacional.

Finalmente, desde la misma concepción de ambientalización curricular (ver Junyent et al., 2008) entendido como un proceso continuo, se deben propiciar espacios de reflexión, planeación y diseño curricular tendientes a mejorar la relación que la comunidad educativa tiene con el recurso ambiental con el que interactúan a diario, desde contenidos que se apoyen en proyectos desarrollados desde las diferentes áreas del conocimiento, los cuales refuercen y promuevan la cultura ambiental sostenible en las diferentes prácticas educativas y sociales de dicha comunidad.

\section{REFERENCIAS}

Arias Rubio, G. (2015). La ambientalización curricular en las humanidades. El caso de la universidad madrileña.

Arriols, E. (22 de 01 de 2018). Ecología Verde. https://www.ecologiaverde.com/que-es-lasostenibilidad-ambiental-y-social1070.html\#anchor_1

Aznar-Minguet, P. , UII, M. A., Martínez-Agut, M. P. , y Piñero, A. (2017). Evaluar para transformar: evaluación de la docencia universitaria bajo el prisma de la sostenibilidad. Enseñanza de las ciencias: revista de investigación y experiencias didácticas, 35(1), 5-27. 
Bedoy, V. (2000). La historia de la educación ambiental: reflexiones pedagógicas. Educar, 13.

Bobbitt, J. F. (1918). The curriculum. Houghton Mifflin.

Bresciano, D., Burger, M., Guevara, R., Martínez Díaz, G., \& Taks, J. (2010). Educación ambiental en la Universidad de la República estado y perspectivas (No. 378: 502 (899) UNle). Universidad de la República (Uruguay).

Brundtland, G. H. (1987). What is sustainable development. Our common future, 8(9).

Burgos, A. (2017). Estado de los proyectos ambientales escolares en Boyacá. Revista Luna Azul, 44 (39).

Caicedo, J., y Calderón, J. (2016). Currículo: en búsqueda de precisiones conceptuales. Revista de Educación y pensamiento. Disponible en: https://dialnet.unirioja. es/ejemplar/445074

Cañal, P. (2011). Competencia científica y competencia profesional en la enseñanza de las ciencias. En Caamaño, A. (coord.). Didáctica de la Física y la Química. Barcelona: Graó-Me, pp. 35-55.

Caswell, H. L., y Campbell, D. S. (1935). Curriculum development. American Book Company. Cátedra, C. M. LA ENSEÑANZA DE TEMAS AMBIENTALES EN LA CARRERA DE INGENIERÍA ELECTROMECÁNICA Y CIVIL EN LA UNIVERSIDAD NACIONAL DEL NORDESTE.

Chablé, G. V., y Delgado, L. T. (2010). Modelos para el diseño curricular. Pampedia, 6, 55-citation_lastpage.

Díaz Barriga, Á. (1985). Didáctica y currículum.

Duque, A. E. (2001). La educación se vuelve industria. https://www.eltiempo.com/archivo/ documento/MAM-509090

Estévez, R. (2013). ¿Qué es una memoria de sostenibilidad? https://www. ecointeligencia. com/2013/09/que-es-una-memoria-desostenibilidad.

Febres-Cordero, M. E., y Florián, D. (2002). Políticas de educación ambiental y formación de capacidades para el desarrollo sustentable. De Río a Johannesburgo. La transición hacia el desarrollo sustentable. Seminario organizado por el PNUMA/INE-SEMARNAT/ Universidad Autónoma Metropolitana. 
Filipe Oliveira, L., Mesa Ortiz, S. L., \& Ruelas Inzunza, E. (2000). Educación ambiental: una guía práctica para profesores, instructores y animadores culturales y de tiempos libres (No. 333.707 O4E3).

Foladori, G. (2000). Contenidos en Educación Ambiental y pensamiento ambientalista. In Ponencia presentada en el III Congreso Iberoamericano de Educación Ambiental.

FRENK, G. A., y CAMPOS, H. G. Educación ambiental y construcción de sociedades sustentables en las sierras veracruzanas.

Gallegos David, A. (2017). Reformas multilaterales sobre comercio y medio ambiente en una economía abierta y contaminada. Estudios Económicos (México, DF), 32(2), 293-315.

Garzón, E. y Acuña, L. (2016). Integración de los proyectos transversales al currículo: una propuesta para enseñar ciudadanía en ciclo inicial. Revista Electrónica "Actualidades Investigativas en Educación", 16(3), 1-26 https://www.redalyc.org/pdf/447/44746861010. pdf

Holguín, M. (2007). Los proyectos transversales escolares y la transversalidad: instrumentos de gestión, dinamización y transformación de la escuela. Revista Interacción, 7 (13), 111 121.

Inlow, G. M. (1966). The emergent in curriculum. Wiley.

Jiménez, M., y Lafuente, R. (2007, September). La conciencia ambiental: qué es y cómo medirla. In Trabajo preparado para el IX Congreso Español de Sociología, grupo de trabajo (Vol. 21).

Junyent, M., Geli, A. M., y Arbat, E. (2003). Ambientalización curricular de los estudios superiores. Girona: Universitat de Girona/Red ACES.

Kitzmann, D. I. S., \& Asmus, M. L. (2012). Ambientalização sistêmica do currículo ao socioambiente.

Martín L. y Bayona H. (2018). Resultados del programa Rectores líderes transformadores en el desarrollo de competencias de los participantes de las Instituciones educativas departamentales (IED) de Cundinamarca. Maestros y maestras investigadores. Ediciones Uniandes 
Mora, P. W. (2012). Ambientalización curricular en la educación superior: un estudio cualitativo en las ideas del profesorado. Profesorado, 16, 79-81.

Novo, M. (1998). La Educación Ambiental a distancia: su alcance y posibilidades. Revista iberoamericana de educación, 16, 101-115.

Oraison, M. (2000). La transversalidad en la educación moral. Foro Iberoamericano de Educación en Valores. OEI. Montevideo.

Parga Lozano, D. L. (2013). Un currículo ambientalizado para formar profesores de Química. Enseñanza de las Ciencias, (Extra), 02662-2666.

Pedraza Jiménez, O. Y. (2017). La interdisciplinariedad en un proceso de ambientalización curricular en la Universidad Pedagógica y Tecnológica de Colombia.

Pierri, N. (2001). Historia del concepto de desarrollo sustentable. Capítulo II (pp. 28781) de Pierri, N. y Foladori, G. (2001). ¿Sustentabilidad? Desacuerdos sobre el desarrollo sustentable. Uruguay: Trabajo y Capital.

Pinar, W. F. (2014). La teoría del currículum (Vol. 132). Narcea Ediciones.

Piñero, A., UII, M. A., Martínez, M. P. , \& Aznar, P. (2007). Introducción de la ambientalización en los currículo de las Ciencias Farmacéuticas. Edusfarm, Revista d'educación superior en Farmacia, 1.

Plata, L. A. M. (2007). Currículo y pertinencia en la educación superior. COOP. EDITORIAL MAGISTERIO.

Sacristán, J. G. (1991). Los materiales y la enseñanza. Cuadernos de pedagogía, (194), 10-15.

Taba, H. (1974). La elaboración del currículo. Troquel.

Velazco, A. (2013, 16 Abril). ¿Qué es el desarrollo sostenible? Blog. https://www.eoi.es/ blogs/mtelcon/2013/04/16/\%C2\%BFque-es-el-desarrollo-sostenible/

Weinstein, C., \& Meyer, D. (1998). Implicaciones de la psicología cognitiva en la aplicación de pruebas: contribuciones a partir del trabajo realizado en estrategias de 
aprendizaje. Wittrock, M. y Baker, E. Test y cognición. Investigación cognitiva y mejora de las pruebas psicológicas. Barcelona: Paidós.

Wood, D. S., \& Wood, D. W. (1990). Cómo planificar un programa de educación ambiental (No. 333.707 W874c). Washington, US: Instituto de Recursos Mundiales, Centro para el Desarrollo Internacional y Medio Ambiente.

Xavier, R. (2007). Curriculum Change and Competencies-Based Approaches: A Worldwide Perspective. 